\title{
High Energy Emissions from the PSR1259-63/SS2883 Binary System
}

\author{
A. Kawachi*, T. Naito ${ }^{\dagger}$ and S. Nagataki** \\ *Institute for Cosmic Ray Research, University of Tokyo, Chiba 277-8582, Japan \\ ${ }^{\dagger}$ Faculty of Management Information, Yamanashi Gakuin University, Yamanashi 400-8575, Japan \\ ** Yukawa Institute for Theoretical Physics, Kyoto University, Kyoto 606-8502, Japan
}

\begin{abstract}
The PSR1259-63/SS2883 binary system consists of a Be star and a radio pulsar in a highly eccentric orbit. We discuss on very-high energy and high energy emissions from the particles accelerated in the Be star flows, due to the interactions of the relativistic pulsar wind and mass outflows of the star. The outflow parameters are constrained by the $\mathrm{TeV}$ observation results.
\end{abstract}

\section{INTRODUCTION}

PSR1259-63 is a radio pulsar which was found to be in a highly eccentric orbit with a 10th magnitude Be star, SS2883 [1]. The orbital period is 1236.7 days and the recent periastron epochs $(\tau)$ were in October 2000 and March 2004. The distance of $1.5 \mathrm{kpc}$ has been estimated from optical photometric observations of SS2883.

The periastron passages have been closely observed at radio frequencies [2,3]. Timing measurements have shown that the disc of the Be star is likely to be inclined with respect to the orbital plane[4]. No pulsed emission was detected for about five weeks centered on periastron. The radio unpulsed light curves around periastron showed two peaks and [3] discussed the results may be caused by the particle acceleration during two shorttime crossings of the pulsar and the disc, before and after periastron. When the pulsar enters the disc, pulsar-wind electrons are accelerated around the contact surface of the pulsar wind and the disc material. After the pulsar leaves the disc, high energy electrons remains in the pulsar-wind bubble. These electrons emits synchrotron radiation in the radio frequencies.

Unpulsed X-ray emission with a single power-law spectrum was detected at the different orbital phases ASCA[5]. The periastron passage in January 1994 was monitored by a multi-wavelength campaign in the X-ray and gamma-ray bands. The power-law spectrum extended to the $200 \mathrm{keV}$ energy region with no pulsations being detected[6]. The result was recently confirmed with INTEGRAL [7]. The hard X-ray emission up to $200 \mathrm{keV}$ strongly implies that it originates from synchrotron radiation of non-thermal electrons. Electrons in the pulsar wind may be accelerated in a shock wave generated in the region where the relativistic pulsar wind interacts with dense mass flow from the $\mathrm{Be}$ star. $[8,9]$ have interpreted the measured hard X-ray spectrum on the basis of accelerated particles in the pulsar-side shock, with Lorentz factor of $\Gamma_{\mathrm{e}}=10^{6}-10^{7}$.

These relativistic electrons in the radiative environment of the binary system may produce high energy gamma rays. Detectable levels of gamma-ray emission was estimated 
from the shocked and un-shocked pulsar wind via inverse Compton scattering [10].

At the contact surface of the pulsar and Be star flows, ions and electrons in the Be star outflow as well may be accelerated to high energies via the first-order Fermi mechanism. In the dense outflow from the Be star there is a lot of target material for proton-proton interactions and Bremsstrahlung emission. The Be star also provides target photons for upscattering by the inverse Compton mechanism, in addition to the $2.7 \mathrm{~K}$ microwave background radiation. The densities of these targets increase as the contact surface gets closer to the Be star, and so does the total energy of accelerated particles. In this paper, a new model calculation for gamma-ray emission from the accelerated particles in the $\mathrm{Be}$ star outflow, taking into consideration Bremsstrahlung, the inverse Compton mechanism, and proton-proton interactions, is applied to the binary system and discussed along with the observational results.

\section{HIGH-ENERGY GAMMA-RAY OBSERVATIONS}

The binary system was observed with the CANGAROO-II Cherenkov telescope at two different orbital phases; for several days in December 2000 and in March 2001, about 47 and 157 days after the periastron of October 2000 [11]. They have no data closer to periastron as observing conditions were not favorable during June-November 2000. The average zenith angles, i.e., the energy thresholds and the effective areas of the observations, were different corresponding to differences in observing seasons. No statistically significant signal of gamma-rays is seen in either of the data sets. The $2 \sigma$ upper limits are of $\sim 0.2-0.5 \mathrm{crab}$ level for the energy threshold of $\sim \mathrm{TeV}$, assuming the spectral index of $-2.0--2.5$.

Though the moon condition was not suitable for a few days including periastron epoch, the observed zenith angles of the target were good at the periastron passage in February to March 2004. The H.E.S.S. system of the 4 Cherenkov telescopes performed observations of the binary system at the periastron passage. With the improved sensitivity and the lower energy threshold of the stereoscopic system, the first sub-TeV gamma-rays from the target were detected in the pre- and post-periastron periods. The signals are about $0.05 \mathrm{crab}$ with the energy threshold of $200 \mathrm{GeV}$ [12].

The CANGAROO-III Cherenkov telescope system (with the three more Cherenkov telescopes additional to the CANGAROO-II) observed it for $\sim 15$ hours in about the same period. The data are under analysis now.

\section{EMISSION MODEL OF THE PARTICLES IN THE BE STAR OUTFLOWS}

A new model of gamma-ray emissivity is introduced, considering the particles accelerated in the Be star outflow [11]. 


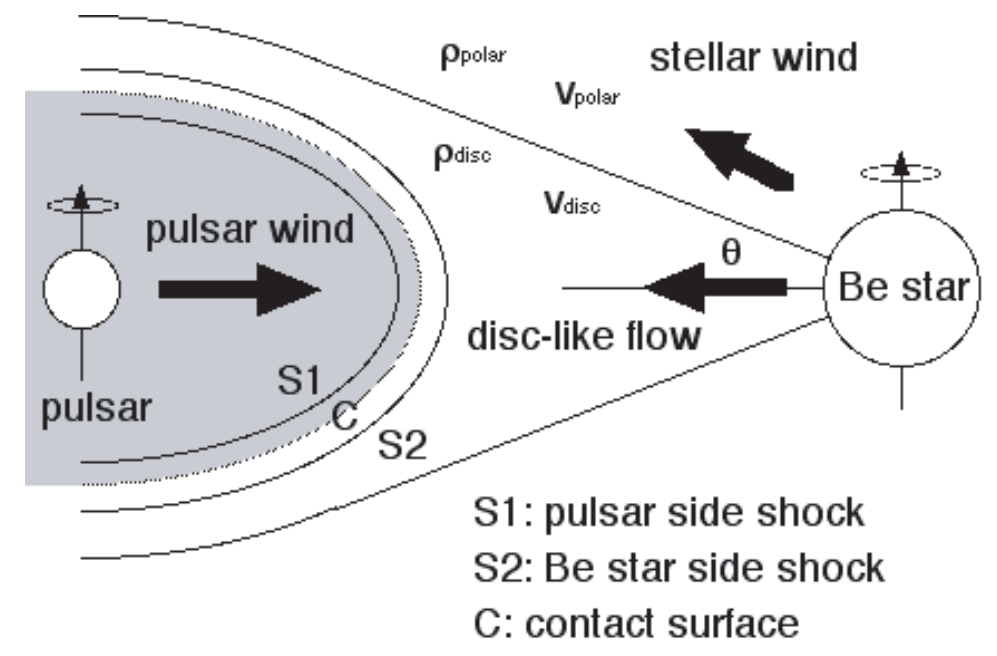

FIGURE 1. Schematic image of the binary system. The wind from the pulsar and the disc-like outflow from the Be star results in a region of pressure balance between the stars.

\section{Description of the model}

Figure 1 schematically illustrates the assumed configuration of the system: the pulsar and its relativistic pulsar wind, the Be star and its polar and disc-like outflows. The shock at the pressure balance between the flows of the two stars composed of three surfaces: pulsar-side shock, contact surface, and Be-star-side shock. Particles are assumed to be accelerated by the shocks. For the pulsar wind, we adopt the model of [13] developed for the synchrotron nebula around the Crab pulsar. For the Be star mass-flow, the simple model of [14] is used, which represents radiations from Be stars using the IR, optical, and UV observational results. The parameters are chosen so as to be consistent with the observational results of Be stars in general [15] and of the PSR B1259-63/SS2883 binary $[1,16,17]$. We fully consider the Klein-Nishina effect in the calculations of the emission processes via electrons.

Provided the pulsar wind is driven by the pulsar spin down luminosity. A fraction $\left(f_{\mathrm{pw}}=0.1\right)$ of the wind luminosity is assumed to be enhanced in the equatorial plane. For the mass-flow of the Be star, we consider a high-density, slow, equatorially orbiting disc-like flow [14], and a low-density, fast, polar component (stellar wind) $[18,19]$ as well. The density profile $\rho(R)$ is assumed to be a power-law (index $n$ ) of the distance from the center of the Be star, $R$, and the flow speed $v(R)$ is obtained from conservation of mass flux. We introduce a new parameter, $x$, defined using $\rho_{0}$ and $v_{0}$ as the density and the velocity of the outflow at the surface of the star, respectively, as

$$
x=\frac{\rho_{0}}{10^{-12} \mathrm{gcm}^{-3}} \frac{v_{0}}{10^{6} \mathrm{cms}^{-1}} .
$$

When $x$ is larger, the location of the pressure balance becomes further from the Be star. The parameter $x$ depends on $v_{0}$ and $\rho_{0}$, which are obtained directly from UV/optical observations, independent of the disc opening angle. As shown later, the gamma-ray 
emission is approximately proportional to $x^{2}$ in our model. The value $x$ of the polar wind component $x_{\text {polar }}$ is set to be proportional to the disc component $x_{\text {disc }}$.

The momentum spectrum of the particles in the Be star outflow are estimated on the basis of Fermi acceleration. For simplicity, we assume that all ions are protons. We assume $f_{\text {acc,i }}=0.001$ and 0.1 is assumed for $i=e$ and $p$, respectively, as the efficiency of the acceleration to be consistent with an e/p ratio in cosmic ray observations (e.g. [20]). The maximum energy of the accelerated particle $E_{\mathrm{e}, \mathrm{p}}^{\mathrm{max}}$ is assumed here to be $\sim 10^{15} \mathrm{eV}$.

Applying the obtained momentum spectra, the gamma-ray spectrum from the source at the distance $D$ is calculated for the emission mechanisms of Bremsstrahlung, inverse Compton (electrons) and proton-proton collisions. The dominant contribution of the calculation is of proton-proton collision. The inverse Compton flux in the sub-TeV energy region, expected from the pulsar-wind side [9], is comparable to that from the Be star outflows, except that the former has a break $\sim 400 \mathrm{GeV}$ due to the stronger magnetic field in the side.

After the spatial integration, the total flux is approximately expressed as

$$
F_{\gamma}\left(E_{\gamma}, x\right) \propto x^{2} \frac{1}{n-1} \frac{1}{R_{\text {shock }}\left(x, n, v_{0}\right)},
$$

where $R_{\text {shock }}$ is the distance to the contact surface from the Be star center. The thick disc-like flow makes the dominant contribution to the total intensity. We investigate 500 $\leq x_{\text {disc }} \leq 5000$ in this model analysis.

\section{Model-calculated light curves}

There are no fixed limits for the orbital phases in which the Be star disc outflow interacts with the pulsar wind. We consider three possibilities in calculating the light curves; (i) aligned disc to the orbital plane and interaction throughout the orbit, (ii) misaligned disc and interaction in the $\sim 200$-day period around periastron $(\tau)$, during which the radio emission is depolarized, or (iii) mis-aligned disc and interaction in two short periods, $[(\tau-18 \mathrm{~d}) \sim(\tau-8 \mathrm{~d})]$ and $[(\tau+12 \mathrm{~d}) \sim(\tau-22 \mathrm{~d})]$, as discussed in [3]. We take account of (i), implying the maximum effect of the disc-pulsar wind interaction, though the disc material becomes dilute at larger distances. The lightcurve with the assumption (iii) is described later in detail.

The CANGAROO observational upper limits [11] are compared with light curves calculated with the model assumption (i) in the disc-pulsar wind interaction. The spectra are integrated $(\mathrm{E} \geq 1 \mathrm{TeV})$ for $n=2.5, v_{0}=10^{6} \mathrm{cms}^{-1}$, and for four different mass outflow parameters, $x_{\text {disc }}=500,1000,1500$, and 5000 (Fig. 2). The outflow parameter is constrained by our results to $x_{\text {disc }} \leq 1500$.

If we simply apply the preliminary report of the H.E.S.S. $\sim 200 \mathrm{GeV}$ observation to our model, the equivalent outflow parameter is $x_{\text {disc }}$ of $\sim 700$. The light curves $(\mathrm{E} \geq$ $200 \mathrm{GeV}$ and $x_{\text {disc }}=700$ ) with the model assumptions (i) and (iii) are shown in Fig. 3. In case of (iii), two peaks corresponds to the twice crossings of the pulsar and the disc. The rise time of emission of the interaction is assumed to be $\sim 1$ day. Between the two peaks, where the pulsar is out of the disc, shock-accelerated particles by the interaction of the 


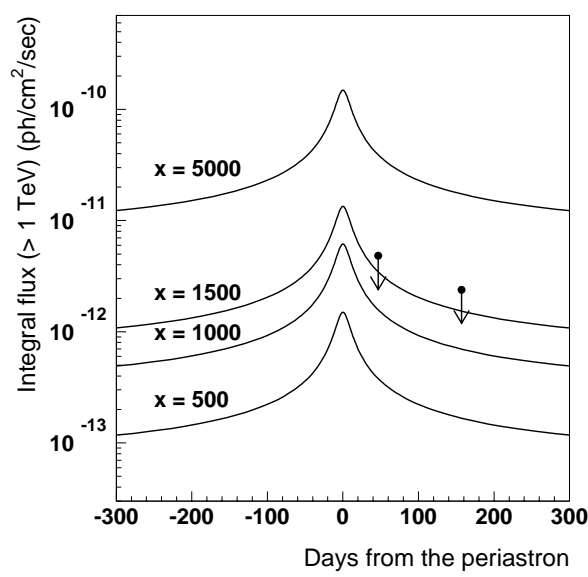

FIGURE 2. Upper limits on the integrated gamma-ray flux are compared with the model calculated curves $(\mathrm{E} \geq 1 \mathrm{TeV})$ as a function of days from periastron epoch. The energy thresholds of our results have been scaled to $1 \mathrm{TeV}$. The light curves of the combined flux from the Be star outflows are calculated with the model assumption (i) (see the text) and $x_{\text {disc }}=500,1000,1500$, and 5000 .

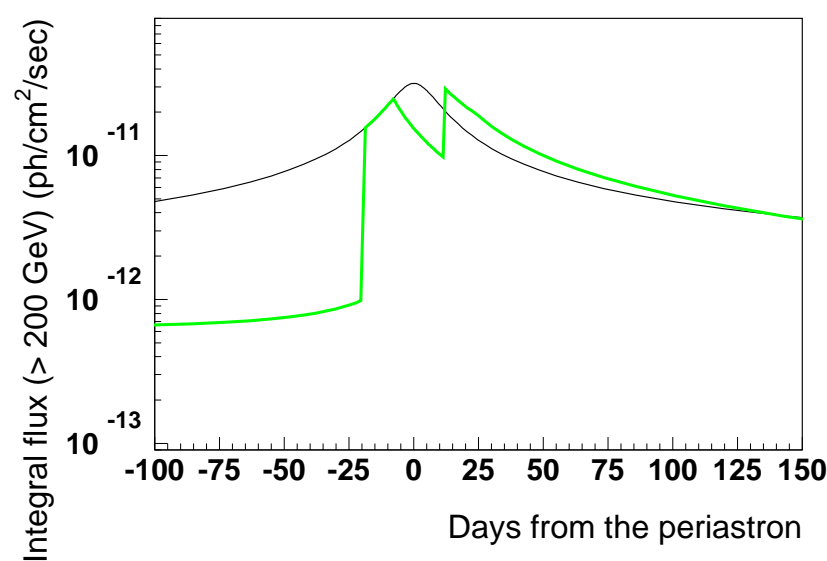

FIGURE 3. The model calculated light curves around the periastron passage (integrated for $\mathrm{E} \geq$ $200 \mathrm{GeV}$ ) with $x_{\text {disc }}=700$ for the different model assumptions (i) and (iii) (see the text).

disc and the pulsar-wind bubble emit the radiation. The emission continues during the second crossing and its contribution to the second peak intensity makes the peak larger than the first one. The intensity decays smoothly after the second crossing ends, as a summed contribution of the first and the second bubbles. The bubble moves outwards in the outflow at the velocity $v_{\text {bubble }}=100 \mathrm{kms}^{-1}$ which is similar to the model in [21] as well as to the typical velocity of the disc flow. According to the radio observations [3], these short-time interaction periods slightly different in the past periastron epochs in different years. 


\section{NEW EMISSION MODEL FOR THE BOTH-SIDE SHOCKS}

A new model framework is being developed to cover emissions from the accelerated particles in the Be-star side and pulsar-side shocks. It includes possible emission mechanisms at the both side shocks and multi-wavelength spectra are calculated to fit the observational results in the wide energy band; from the infrared to the latest detection of the high-energy gamma-ray [22]. A preliminary result shows that radio and high-energy gamma-ray emissions are mainly of the Be-star side while X-ray emissions are of the pulsar side.

\section{SUMMARY}

We discuss on high-energy emissions of the PSR1259-63/SS2883 binary focusing on the particles accelerated in the Be star outflows due to the interactions of the relativistic pulsar wind and mass outflows. A new model framework is being studied to include emissions of the pulsar-wind side.

\section{REFERENCES}

1. Johnston, S., et al., Mon. Not. R. Astron. Soc., 268, 430 (1994).

2. Johnston, S., et al., Mon. Not. R. Astron. Soc., 302, 277 (1999).

3. Connors, T. W., et al., Mon. Not. R. Astron. Soc., 336, 1201 (2002).

4. Wex, N., et al., Mon. Not. R. Astron. Soc., 298, 997 (1998).

5. Hirayama, M., et al., Atrophys. J., 521, 718 (1999).

6. Grove, J. E., et al., Atrophys. J., 447, L113 (1995).

7. Shaw, S. E., et al., astro-ph/0409253 (2004).

8. Tavani, M., and Arons, J., Atrophys. J., 477, 439 (1997).

9. Murata, K., et al., Pub. Astron. Soc. Japan, 55, 467 (2003).

10. Ball, L., and Kirk, J. G., Astropart. Phys., 12, 335 (2000).

11. Kawachi, A., et al., Atrophys. J., 607, 949 (2004).

12. Beilicke, M., et al., IAUC 8300 (1984).

13. Kennel, C. F., and Coroniti, F. V., Atrophys. J., 283, 694 (1984).

14. Waters, L. B. F. M., et al., Astron. Astrophys., 162, 121 (1986).

15. Cote, J., and Waters, L. B. F. M., Astron. Astrophys., 176, 93 (1987).

16. Johnston, S., et al., Mon. Not. R. Astron. Soc., 279, 1026 (1996).

17. Melatos, A., et al., Mon. Not. R. Astron. Soc., 275, 381 (1995).

18. Waters, L. B. F. M., et al., Astron. Astrophys., 198, 200 (1988).

19. Dougherty, S. M., et al., Astron. Astrophys., 290, 609 (1994).

20. Baring, M. G., et al., Atrophys. J., 513, 311 (1999).

21. Paredes, J. M., et al., Astron. Astrophys., 248, 124 (1991).

22. Nagataki, S., et al., in preparation (2004). 